\title{
Joint-specific assessment of swelling and power Doppler in obese rheumatoid arthritis patients
}

\author{
Erin M. Bauer ${ }^{1,2}$, Ami Ben-Artzi ${ }^{3}$, Erin L. Duffy', David A. Elashoff', Sitaram S. Vangala', John Fitzgerald \\ and Veena K. Ranganath ${ }^{*}$
}

\begin{abstract}
Background: Clinical swollen joint examination of the obese rheumatoid arthritis (RA) patient can be difficult. Musculoskeletal Ultrasound (MSUS) has higher sensitivity than physical examination for swollen joints (SJ). The purpose of this study was to determine the joint-specific association between power Doppler (PDUS) and clinical SJ in RA across body mass index (BMI) categories.

Methods: Cross-sectional clinical and laboratory data were collected on 43 RA patients. PDUS was performed on 9 joints (wrist, metacarpalphalangeal 2-5, proximal interphalgeal 2/3 and metatarsalphalangeal 2/5). DAS28 and clinical disease activity index (CDAl) were calculated. Patients were categorized by BMI: $<25,25-30$, and $>30$. Demographic and clinical characteristics were compared across BMI groups with Kruskal-Wallis test and chi-square tests. Joint-level associations between PDUS and clinically SJ were evaluated with mixed effects logistic regression models.

Results: While demographics and clinically-determined disease activity were similar among BMI groups, PDUS scores significantly differed $(p=0.02)$. Using PDUS activity as the reference standard for synovitis and clinically SJ as the test, the positive predictive value of SJ was significantly lower in higher BMl groups ( 0.71 in $\mathrm{BMl}<25,0.58$ in $\mathrm{BMI}$ $25-30$ and 0.44 in $\mathrm{BMI}<30)(p=0.02)$. The logistic model demonstrated that increased BMl category resulted in decreased likelihood of PDUS positivity (OR 0.52, $p=0.03$ ).

Conclusions: This study suggests that in an obese RA patient, a clinically assessed SJ is less likely to represent true synovitis (as measured by PDUS). Disease activity in obese RA patients may be overestimated by CDAI/ DAS28 calculations and clinicians when considering change in therapy.
\end{abstract}

Keywords: Ultrasound, Obesity, Outcome measures, Rheumatoid arthritis

\section{Background}

Musculoskeletal ultrasound (MSUS) with power Doppler (PDUS) has become an accepted modality to identify features of inflammatory arthritis including synovitis and enthesitis and expert panels from the American College of Rheumatology (ACR) and the European League Against Rheumatism (EULAR) consider its use reasonable for monitoring disease activity in patients with rheumatoid arthritis (RA) [1, 2]. Synovitis is the manifestation of synovial proliferation and angiogenesis, which is an early

\footnotetext{
*Correspondence: vranganath@mednet.ucla.edu

'Department of Medicine, David Geffen School of Medicine, University of

California, Los Angeles, UCLA, Los Angeles, CA, USA

Full list of author information is available at the end of the article
}

pathologic change leading to bony destruction by locally invading the synovium-cartilage interface [3]. The Outcome Measures in Rheumatoid Arthritis Clinical Trials (OMERACT) initiative defines synovitis by PDUS as abnormal hypoechoic intraarticular tissue that is non-displaceable, poorly compressible, and exhibiting Doppler signal [4]. Unlike healthy synovium, inflamed synovium is hyperemic and can be semi quantitatively graded, most commonly on a 4-point scale of 0 to 3 for PDUS activity [5]. Multiple studies measuring PDUS have documented improvement of PDUS scores in response to RA treatments [6, 7]. PDUS is reported to have higher sensitivity and be more reliable for synovitis evaluation than an examination of swollen 
joints [8] and is predictive of relapse and radiographic disease progression [9].

While the uses and benefits of MSUS in RA are becoming widely recognized, the burden of time and cost can be prohibitive in obtaining MSUS on every RA patient within the rheumatologists' clinical practice. It may be beneficial to better define the type of RA patient and clinical scenario in which MSUS will have differential impact on therapeutic management especially given the push towards early and aggressive control of disease activity. The majority of RA patients in the United States are considered overweight or obese $[10,11]$, and these patients have a poorer quality of life [12] and are less likely to attain remission by Disease Activity Score (DAS28) $<2.6$ despite treatment as compared to non-obese RA patients [13, 14]. This lack of measured response in obese RA patient could be due to a differential response to therapy, a measurement error in assessing disease activity, or a combination of both. PDUS in the obese RA patient has not been fully described in the literature to date.

Our objective in this study was to evaluate the joint specific association between synovitis measured by PDUS and the clinically swollen joint in overweight and obese RA patients.

\section{Methods}

\section{Patients}

Patients meeting the ACR 1987 and ACR/EULAR RA diagnostic criteria $[15,16]$ and whose treating physicians were considering switching and/or escalating immunosuppressive therapy were recruited from University of California at Los Angeles (UCLA) rheumatology clinics. Patients were required to have active RA, with a minimal swollen joint count (SJC) $\geq 2$ and tender joint count (TJC) $\geq$ 2 of the 28 joint count examined. Eligible RA patients met the following criteria: age $\geq 18$, stable disease modifying anti-rheumatic drugs (DMARDs), and on prednisone $\leq$ $10 \mathrm{mg}$ for at least one month. Patients were excluded if they were pregnant or breast feeding, or if they had arthroplasty of the joints examined by ultrasound. Based on these inclusion/exclusion criteria, 43 patients were evaluated. These patients were then divided into three groups based on their body mass index (BMI): $<25,25$ to 30 , and $>30$ for analysis. The study was approved by the UCLA institutional review board (IRB\#11-001225-CR-00005).

\section{Clinical study measures}

Patients completed demographic questionnaires, and the components of the DAS28 and Clinical Disease Activity Index (CDAI) were obtained the same day as the ultrasound. The erythrocyte sedimentation rate (ESR) was measured to calculate the DAS28/ESR. Rheumatoid factor (RF) and anti-cyclic citrullinated protein antibody
(ACPA) were obtained for each patient. The total tender and swollen joint count was assessed by a single study investigator (VKR) prior to ultrasound.

\section{Musculoskeletal ultrasound synovitis measures}

MSUS assessment of PDUS and grey scale (GSUS) hypertrophy and effusion was performed at the onetime visit. The sonographer (ABA) was an experienced rheumatologist trained in MSUS and certified in Rheumatology MSUS (rhMSUS). Images were obtained using a GE Logic E9 machine with ML6-15 linear probe and the following presets: red-yellow color map, Doppler frequency $10.0 \mathrm{MHz}$, PRF $0.8 \mathrm{KHz}$, and gain adjusted just below noise. Based on a prior publication by Backhaus et al [6], with the addition of 2 joints (metacarpalphalangeal 4 and 5), a total of 9 joints were scanned by MSUS on the most active side: wrist (dorsal longitudinal midline view), metacarpalphalangeal (MCP) joints 2, 3, 4, and 5 (dorsal/palmar long views), proximal interphalgeal (PIP) joints 2 and 3 (dorsal/palmar long views) and metatarsalphalangeal joints 2 and 5 (dorsal long view). PDUS and GSUS were scored semi-quantitatively on a scale of $0-3$ per prior published consensus definitions [6]. Specifically, PDUS was scored by the amount of power Doppler signal in the intraarticular area: grade $0=$ no color signal, grade $1=$ up to 3 color signals or 2 single and 1 confluent signal, grade $2=$ greater than grade 1 to $<50 \%$ color signal, and grade $3=>50 \%$ color signal [6]. The max score of the views obtained for each joint was computed and then was theses maximums were summed across all 9 joints to obtain total PDUS (range 0-27) and GSUS (range $0-27)$ scores. An individual joint was considered to be positive for synovitis if its max PDUS score was $\geq 1$. The clinical assessor was blinded to the ultrasound data, and the ultrasonographer was blinded to the clinical assessments. Additionally, when scoring the archived images, the ultrasonographer was blinded to all clinical data. Once all scans were completed the ultrasonographer re-read $10 \%$ of randomly selected ultrasound visits for kappa intra-rater reliability calculations for GSUS and PDUS [17].

\section{Statistical analysis}

Patients' mean (standard deviation [SD]) age and disease duration were calculated for each BMI group and compared across groups with $\mathrm{t}$-tests. The proportion of female and sero-positive $(\mathrm{RF}+$ or $\mathrm{ACPA}+)$ patients was calculated for each BMI group and compared across groups with Fisher Exact Tests. The GSUS, PDUS, SJC, TJC, DAS28/ ESR-4, CDAI, and ESR for each BMI group were compared across BMI groups with Kruskal-Wallis tests. Kruskal-Wallis Tests were used for these US and clinical measures because they exhibited skewed distributions in visual assessments. 
Agreement between clinical swollen joint assessments and PDUS assessments were evaluated for the full cohort and each BMI group in several ways. First, the overall percent of joints that were considered swollen and PDUS positive were calculated for the full cohort and each BMI group. Next, the percent of joint-specific pairwise agreement between swollen joint assessment and PDUS assessment was calculated. To assess the predictive relationship of clinical swollen joints versus PDUS positivity (reference standard), the sensitivity, specificity, positive predictive value (PPV), and negative predictive value (NPV) of swollen joint assessment were calculated.

We constructed a mixed effects logistic regression model to evaluate the correlates of PDUS positivity, as well as for GSUS. This model included terms for SJC, BMI group (represented ordinally), age, sex, joint, as well as a random effect for subject to account for the clustering of joints within subjects. Within the logistic regression, BMI was represented as an ordinal variable by converting the 3 categories of BMI as follows: $\mathrm{BMI}<25=1$, BMI 25-30 = 2, and $\mathrm{BMI}>30=3$. To evaluate the predictive ability of this model for PDUS positivity, we constructed a ROC curve using the predicted probabilities from the model and computed the area under the ROC curve (AUC). Lastly, we included a term in the model for specific joint to evaluate in which joints the differences between BMI groups were most pronounced.

\section{Results}

Demographic characteristics were similar among BMI groups. The overall cohort was $86 \%$ female and $66 \%$ ACPA or RF seropositive with a mean age of 52.1 (SD 12.9) and disease duration of 7.3 (SD 7.8) years (Table 1). Within the cohort $93 \%$ of patients were on a DMARD, $19 \%$ were on Biologics, and $16 \%$ of patients were on prednisone. There were no significant differences in these characteristics across the BMI categories.

The overall median and interquartile ranges (IQR): 4 (2) for SJC, 4 (4) for TJC, 6.3 (1.5) for DAS28/ESR-4, and 36.5
(29.0) CDAI score (Table 2). These disease activity as measures were not significantly different across the BMI groups. While GSUS was not significantly different across BMI groups $(p=0.42)$, the PDUS scores significantly differed across BMI groups with median (IQR) scores of 3

(3) for BMI group <25, 1 (3.5) for BMI group 25-30 and 0

(2) BMI group $>30(p=0.02)$.

The prevalence of PDUS positive joints decreased across BMI categories (54\% for BMI $<25$, 40\% for BMI 25-30, and 30\% for BMI > 30), while prevalence of swollen joints in the high BMI group was only 3 percentage points lower than in the low BMI group ( $48 \%$ for $\mathrm{BMI}<25$ vs $45 \%$ for BMI > 30) (Table 3). Using PDUS activity as the reference standard for synovitis and SJC as the test (jointspecific analyses), the positive predictive value (PPV) of SJC was significantly lower in higher BMI groups (0.71 in $\mathrm{BMI}<25,0.58$ in BMI 25-30 and 0.44 in BMI $>30$, $p=0.02)$. The negative predicative value (NPV) trended higher in $>30 \mathrm{BMI}$ group $(0.62$ for BMI group $<25$, 0.71 for BMI group 25-30, 0.81 in BMI group >30) although was not statistically significant. In addition, clinically swollen joints had higher rates of PDUS compared to non-swollen joints, and there was a decreasing trend of PDUS positivity with increasing BMI for both swollen and non-swollen joints (Fig. 1). For example, a clinically swollen joint in a RA patient with normal BMI had 71\% chance of PDUS positivity, whereas a swollen joint in an obese patient had a $44 \%$ chance. On the other hand, a non-swollen joint in a normal BMI patient still had a PDUS positivity rate of $38 \%$, while lower in the obese patient's non-swollen joint (19\%).

The logistic regression model demonstrates that each BMI category increase is independently associated with lower odds of PDUS positivity with an odds ratio of 0.52 (95\% CI: $0.08,0.88, p=0.03$ ), adjusting for covariates (Table 4). Additionally, the logistic model shows that the presence of clinical joint swelling is positively associated with the likelihood of PDUS positivity $(\mathrm{OR}=2.5,95 \% \mathrm{CI}$ : 1.4 to $4.5, p=0.01)$. The AUC of the logistic model was

Table 1 Baseline demographics, mean (SD) or number (\%)

\begin{tabular}{|c|c|c|c|c|c|c|c|c|}
\hline \multirow[b]{3}{*}{$\overline{\mathrm{BMI}^{1, *}}$} & \multirow{3}{*}{$\begin{array}{l}\text { Overall } \\
N=43 \\
28.9(9.0)\end{array}$} & \multirow{2}{*}{\multicolumn{2}{|c|}{$\begin{array}{l}\mathrm{BMI}<25 \\
N=17\end{array}$}} & \multirow{2}{*}{\multicolumn{2}{|c|}{$\begin{array}{l}\mathrm{BMI} 25-30 \\
N=12\end{array}$}} & \multirow{2}{*}{\multicolumn{2}{|c|}{$\begin{array}{l}\mathrm{BMI}>30 \\
N=14\end{array}$}} & \multirow[t]{3}{*}{$P$-value } \\
\hline & & & & & & & & \\
\hline & & 21.3 & $(2.3)$ & 27.2 & $(1.4)$ & 39.5 & $(7.3)$ & \\
\hline Age (years)* & $52.1(12.9)$ & 49.9 & $(16.9)$ & 56.6 & $(7.5)$ & 51.1 & $(10.5)$ & 0.37 \\
\hline Disease duration (years)* & $7.3(7.8)$ & 7.4 & $(9.6)$ & 4.0 & $(3.8)$ & 10.1 & $(7.2)$ & 0.14 \\
\hline Female** & $37(86 \%)$ & 17 & $(100 \%)$ & 8 & $(67 \%)$ & 12 & $(86 \%)$ & 0.02 \\
\hline $\mathrm{RF}^{2}$ or $C C P^{3}$ Positive ${ }^{* *}$ & $27(66 \%)$ & 11 & $(69 \%)$ & 9 & $(75 \%)$ & 7 & $(54 \%)$ & 0.55 \\
\hline $\operatorname{DMARD}(Y)$ & $40(93 \%)$ & 1 & $(6 \%)$ & 0 & $(0 \%)$ & 2 & $(14 \%)$ & 0.48 \\
\hline Biologics $(Y)$ & $8(19 \%)$ & 2 & $(12 \%)$ & 2 & $(17 \%)$ & 4 & $(28 \%)$ & 0.55 \\
\hline Prednisone $(Y)$ & 7 (16\%) & 3 & $(18 \%)$ & 2 & $(17 \%)$ & 2 & $(14 \%)$ & 0.99 \\
\hline
\end{tabular}

${ }^{1}$ Body Mass Index, ${ }^{2}$ Rheumatoid Factor, ${ }^{3}$ Cyclic citrullinated peptide, ${ }^{*}$ Mean (SD) and one-way ANOVA, ${ }^{* *} \mathrm{~N}(\%)$ and Fisher Exact Test 
Table 2 Musculoskeletal ultrasound synovitis measures and disease activity measures, median IQR (25\%, 75\%)

\begin{tabular}{|c|c|c|c|c|c|c|c|c|}
\hline & Overall & BMI & & BMI & & $\mathrm{BMI}$ & & $P$-value \\
\hline & $N=43$ & $N=$ & & $N=$ & & $N=$ & & \\
\hline SJC & $4(3,5)$ & 5 & $(3,5)$ & 4 & $(2,4.5)$ & 4 & $(3,5)$ & 0.40 \\
\hline TJC & $4(2,6)$ & 4 & $(3,6)$ & 3 & $(2,5)$ & 4 & $(2,6)$ & 0.57 \\
\hline ESR & $36.5(25,54)$ & 37 & $(30,54)$ & 35 & $(24,52)$ & 40 & $(25,54)$ & 0.77 \\
\hline DAS28/ESR-4 & $6.3(5.4,6.9)$ & 6.6 & $(5.4,7.0)$ & 6.2 & $(5.5,6.5)$ & 6.1 & $(5.2,6.9)$ & 0.44 \\
\hline CDAl & $36(26,43)$ & 36 & $(30,44)$ & 35 & $(21.5,42)$ & 35 & $(26,42)$ & 0.67 \\
\hline GSUS & $5(3,6)$ & 5 & $(3,6)$ & 4 & $(2.5,6)$ & 5.5 & $(4,6)$ & 0.42 \\
\hline PDUS & $1(0,3)$ & 3 & $(1,4)$ & 1 & $(0,3.5)$ & 0 & $(0,2)$ & 0.02 \\
\hline
\end{tabular}

GSUS-Gray Scale Ultrasound, PDUS-Power doppler Ultrasound, SJC-Swollen Joint Count, TJC-Tender Joint Count, CDAI-Clinical Disease Activity Index, ESR-Erythrocyte Sedimentation Rate. Kruskal-Wallis tests used to compare groups

0.76 suggesting the combination of BMI, clinical swollen joint assessment, sex and age provide strongly predict the presence of PDUS positivity. Additionally, we constructed a similar logistic model for outcome of GSUS positivity and found similar results for the association of clinical swelling and GSUS $(\mathrm{OR}=2.52)$ to what was observed in the PDUS model (Table in Additional file 1). In particular, the wrist, MCP2, and MCP3 had significantly less PDUS positivity in the BMI $>30$ group as compared to the $\mathrm{BMI}<25$ group (Fig. 2). The ultrasonographer's intra-rater reliability for GSUS and PDUS was a kappa of 0.62 and 0.82 , respectively.

\section{Discussion}

Despite continuing advances in therapeutic options for RA, almost $30 \%$ of all RA patients will fail to meet the goals of quick and sustained control of disease activity with a first exposure to a biologic agent and will need to switch therapy [18]. Given the emphasis on early intervention to prevent the destructive changes in RA and the push to "treat to target" (T2T) by national and international guidelines [19-21], there is incentive to know when a patient is not responding to a given treatment. The current gold standard for monitoring disease activity and thus the "target" of T2T is usually DAS28 remission $(<2.6)$ or low activity $(<3.2)$. While the majority of T2T studies and guidelines employ DAS28, CDAI, or Simple Disease Activity Index (SDAI), there are

Table 3 PDUS and Swollen Joint Agreement by BMI Group

\begin{tabular}{llllll}
\hline & Overall & $\mathrm{BMI}<25$ & $\mathrm{BMI} 25-30$ & $\mathrm{BMI}>30$ & $p$ \\
\hline \% Joints PDUS Positive & $42 \%$ & $54 \%$ & $40 \%$ & $30 \%$ & 0.018 \\
\% Joints Swollen & $44 \%$ & $48 \%$ & $37 \%$ & $45 \%$ & 0.611 \\
\% Agreement & $66 \%$ & $66 \%$ & $66 \%$ & $64 \%$ & 0.713 \\
Sensitivity & 0.61 & 0.64 & 0.53 & 0.66 & 0.822 \\
Specificity & 0.68 & 0.70 & 0.74 & 0.64 & 0.691 \\
PPV & 0.59 & 0.71 & 0.58 & 0.44 & 0.023 \\
NPV & 0.71 & 0.62 & 0.71 & 0.81 & 0.084 \\
\hline PPV Positive Predictive Value, NPV Negative Predictive Value &
\end{tabular}

PPV Positive Predictive Value, NPV Negative Predictive Value criticisms that these targets require prior blood work (DAS28, SDAI), do not account for the joints in the feet, and that the tender joint counts can be misleading for disease activity measures due to attribution error in patients with co-morbidities such as fibromyalgia and osteoarthritis. Our study addresses the latter point. Other studies have suggested that RA patients with fibromyalgia were found to have elevated disease activity scores, however noted to have low or absent measures of clinical synovitis or MSUS synovitis [22, 23]. Our study is the first to our knowledge, which investigates the discordance of elevated disease activity scores in obese patients and absent/low measureable synovitis by PDUS.

The obese and overweight patients with RA pose a considerable management challenge. Studies report that obese and overweight patients with RA are less likely to attain disease remission [24-26] and are more likely to have limited therapeutic response [13] as compared to non-obese RA patients, even with weight-adjusted treatments [14]. Sandberg et al found a significant dose response relationship between a BMI and change in disease activity in a study of 495 Swedish patients. Those with a $\mathrm{BMI} \geq 25$ had a $51 \%$ lower odds of reaching low disease activity as measured by DAS28 after 6 months of follow up ( $\mathrm{OR}=0.4995 \% \mathrm{CI} 0.31$ to 0.78$)$ [18]. Obese RA patients also have higher rates of functional disability, cardiovascular risks, and decreased quality of life as compared to non-obese RA patients [27, 28]. Interestingly, although overweight patients have a decreased chance of achieving good disease control as measured by DAS28, multiple studies have suggested that patients with higher BMIs may have less radiographic joint damage [29], in particular in ACPA positive obese patients [30]. Thus, the natural question that arises is whether there could be a potential measurement error of disease activity in obese RA.

Our study implies that clinically assessed swollen joints are less likely to represent true synovitis in obese RA patients. Therefore, in obese patients, RA disease activity can be overestimated by CDAI and DAS28 


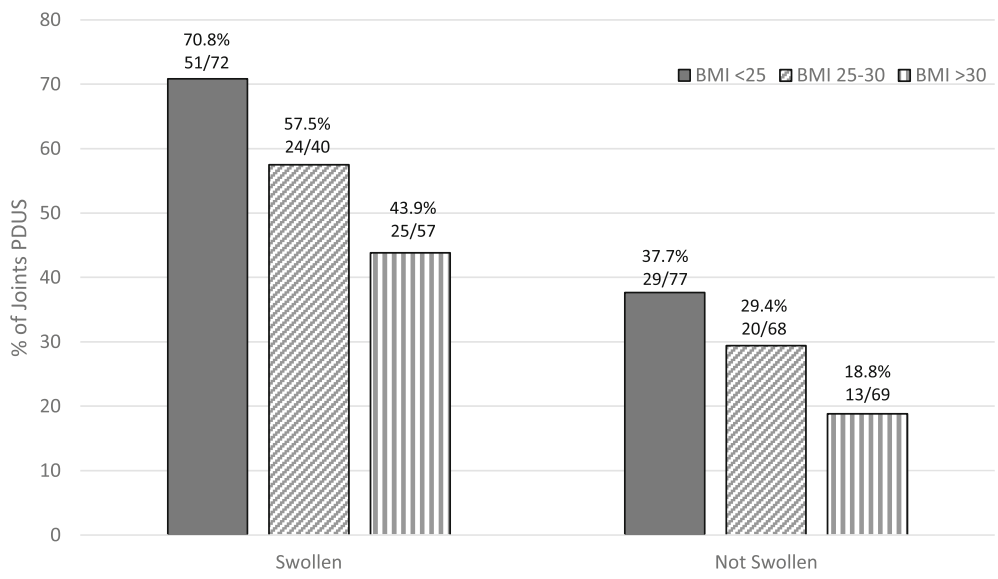

Fig. 1 The proportion of PDUS positivity stratified by BMI category and swollen joint status. PDUS: power Doppler ultrasound, BMI: body mass index

calculations which may help to explain the reports that obese and overweight patients with RA are less likely to attain remission and are more likely to have limited therapeutic response as compared to non-obese RA patients.

Multiple studies have reported that increased BMI is associated with less radiographic joint damage (total erosions, joint space narrowing) in RA [29-31]. Theories to explain this have invoked the protective effects of adipocytes on synovial tissue, decreased hydroxypyridinium collagen crosslinking, the anti-inflammatory properties of adiponectin and estrogen [32] and beneficial bone remodeling secondary to greater weight $[29,33]$. Our study suggests that obese RA patients may simply have less synovitis as compared to non-obese patients with the same calculated disease activity scores. This is an alternate possible explanation for prior observations that obese patients who have a less severe disease course radiographically as compared to non-obese matched controls. The assumption that joint destruction in RA is related to synovial hyperemia is supported by several studies including a 2008 study by Naredo et al which included MSUS with PDUS of 367 patients with RA and demonstrated that PDUS signal was predicative of radiographic erosions and progression on plain films $(\mathrm{R}=0.64)$ [34]. In addition, studies using bone marrow edema as seen on magnetic resonance imaging (MRI) to assess for disease activity have shown similar results. In a

Table 4 Multivariate Logistic model for PDUS (accounts for correlation among different joints in the same patient)

\begin{tabular}{lcccc}
\hline & Odds Ratio & 95\% Cl Lower & 95\% Cl Upper & $P$-value \\
\hline Age & 1.01 & 0.98 & 1.05 & 0.48 \\
Sex (Female) & 2.42 & 0.54 & 10.87 & 0.25 \\
BMI (Ordinal) & 0.52 & 0.30 & 0.93 & 0.03 \\
Swollen (Yes) & 2.50 & 1.40 & 4.48 & 0.01 \\
\hline
\end{tabular}

PDUS power Doppler ultrasound
2014 Baker et al study, a secondary analysis of the GO-BEFORE and GO-FORWARD randomized clinical trials, evaluated the efficacy of golimumab in combination with methotrexate compared with methotrexate and golimumab monotherapy and demonstrated that there was a significantly negative correlation between bone edema score and BMI at baseline [33].

Both ACR and EULAR support the use of MSUS to monitor RA disease activity and this study suggests that it may be a particularly important tool in the evaluation and management of the obese patient. In the United States $60-70 \%$ of RA patients are overweight or obese $[10,11]$. Therefore, assessment of PDUS could be considered in the majority of our RA patients in order to gain a better understanding of their true disease activity. Prior studies have suggested that MSUS with PDUS is more sensitive than physical examination in detection of synovitis [8] which is consistent with our study as obese RA subjects with clinically swollen joints have a lower PPV and a trend for higher NPV for true synovitis, while the opposite is found for normal BMI patients.

Frequent MSUS assessments require an experienced and consistent ultrasonographer and is a time consuming process if a large number of joints are examined. Recent studies have suggested that the addition of MSUS to management of an early RA patient population provides no additional effect compared with a conventional tight control strategy $[35,36]$. However, if the correct established RA patient population can be targeted, the costs of MSUS may off set the burdens associated with switching/escalating pharmacologic therapies including support/authorization staff time, adverse events and side effects of immunosuppressive medications. Our study suggests that of all the joints included, the wrist, MCP2 and MCP 3 show the greatest discrepancy in swollen joint count by exam and PDUS activity between patients with $\mathrm{BMI}>30$ and patients with 


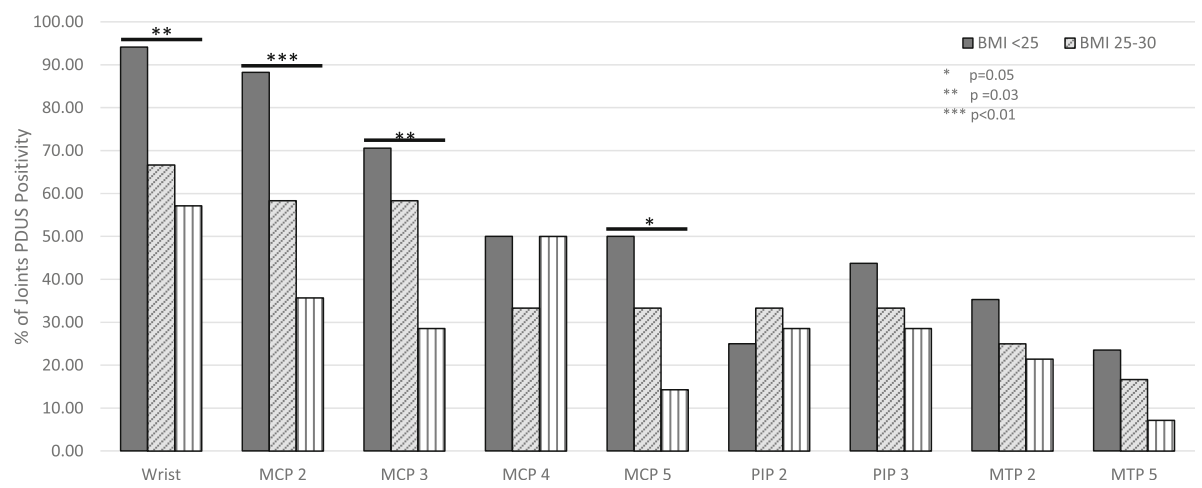

Fig. 2 Percentage of PDUS positivity stratified by joint and BMI category

BMI $<25$. Further study could lead to the ability to perform a more targeted exam.

This study is limited by a relatively small sample size, however, several of our analyses included a larger number of joint-specific pairwise agreement assessments between swollen joint and PDUS. While the baseline patient characteristics were similar across all BMI groups there were no men in the $\mathrm{BMI}<25$ group. This was a cross-sectional study and thus we are unable to examine the differential effect, if any, on treatment response in patients who are overweight or obese. Other patient co-morbidities such as osteoarthritis and fibromyalgia were not obtained. Aside from ESR and CRP we did not measure markers that others have correlated with obese patients such as adiponectin and leptin. We chose MSUS with PDUS as our gold standard for synovitis while some may consider MRI the gold standard. However, MRI is limited in the number of joints that can be assessed and requires contrast and, studies have demonstrated a high level of agreement between MSUS with PDUS and MRI for detection of synovitis [37, 38] with at least one study reporting that US was more sensitive than MRI in the detection of synovitis [39]. Additionally, as mentioned above, there is evidence that obese patients have significantly less synovitis as measured by RA MRI (RAMRIS) synovitis score as compared to patients with BMI $<30$ despite having similar disease activity scores measured by DAS28(CRP) which is consistent with our findings [33]. Lastly, we noted there was a trivial difference in the depth from skin to joint in the obese patients, but only at the wrist $(0.1 \mathrm{~mm})$, versus those patients who were overweight or normal. This difference was not significant enough to explain lower scores (by way of beam penetration) in the obese patients.

\section{Conclusion}

This study suggests that in a cohort of RA patients whose rheumatologist was considering switching therapy, obese RA subjects had lower PDUS scores than non-obese patients while having similar clinical disease activity scores. In addition, $\mathrm{BMI} \geq 30$ was an independent predictor of lower PDUS while accounting for other factors. Obese RA patients' clinically swollen joints had a lower PPV and a higher NPV for true synovitis. This implies that clinically assessed swollen joints are less likely to represent true synovitis in obese RA patients, suggesting that RA disease activity can be overestimated by CDAI and DAS28 calculations. With the push towards "treating to target" of remission or low disease activity by DAS28 or CDAI alone, the BMI of the patient should taken in to account. If there is a question of whether a joint is swollen, MSUS with PDUS could be considered prior to changing or escalating therapy.

\section{Additional file}

Additional file 1: Obesity US Manuscript Supplemental Material. Multivariate Logistic model for GSUS (accounts for correlation among different joints in the same patient). (DOCX $12 \mathrm{~kb}$ )

\section{Abbreviations}

ACPA: Anti-cyclic citrullinated protein antibody; ACR: American College of Rheumatology; BMI: Body mass index; CDAl: Clinical disease activity index; DAS: Disease Activity Score; DMARD: Disease modifying anti-rheumatic drug; ESR: Erythrocyte sedimentation rate; EULAR: European League Against Rheumatism; GSUS: Grey scale synovial hypertrophy; IQR: Interquartile range; MCP: Metacarpalphalangeal; MSUS: Musculoskeletal ultrasound; NPV: Negative predictive value; OMERACT: Outcome Measures in Rheumatoid Arthritis Clinical Trials; PDUS: Power Doppler ultrasound; PIP: Proximal interphalgeal; PPV: Positive predictive value; PPV: Positive predictive value; RA: Rheumatoid arthritis; RF: Rheumatoid factor; SD: Standard deviation; SDAl: Simple Disease Activity Index; SJ: Swollen joints; SJC: swollen joint count; T2T: Treat to target; TJC: Tender joint count; UCLA: University of California at Los Angeles.

\section{Acknowledgments}

Cesar Olmos provided significant assistance in the collection of data.

\section{Funding}

This work was supported by Grant and National Institutes of Health Award (NIAMS K23 AR057818-02) to Veena K. Ranganath and supported by the National Center for Advancing Translational Sciences through UCLA CTSI Grant UL1TR000124. 


\section{Availability of data and materials}

We will share data with any investigator interested in replicating these findings or interested in future collaborations, pursuant to institutional and IRB regulations, in accordance with patient privacy, confidentiality.

\section{Authors' contributions}

EMB drafted and revised the manuscript, analyzed and interpreted the data. VKR revised the manuscript, made substantial contributions to conception and design of the study, acquisition of data and interpretation of data. ABA made substantial contributions to acquisition of data and design of the study. ELD, SV, and DAE provided statistical analysis and interpretation of data. JDF made substantial contributions to analysis and interpretation of data as well as critically revising the manuscript for important intellectual content. All authors have approved the manuscript for submission, and have given necessary attention to ensure the integrity of the work. We confirm that each individual named as an author meets the Uniform Requirements for Manuscripts Submitted to Biomedical Journals criteria for authorship.

\section{Competing interests}

The authors declare that they have no competing interests.

\section{Consent for publications}

\section{Not applicable.}

\section{Ethics approval and consent to participate}

This study was approved by the University of California Los Angeles' research ethics board (IRB\#11-001225-CR-00005) and written informed consent was obtained from all participants.

\section{Author details}

'Department of Medicine, David Geffen School of Medicine, University of California, Los Angeles, UCLA, Los Angeles, CA, USA. ${ }^{2}$ U.S. Department of Veterans Affairs, Los Angeles, CA, USA. ${ }^{3}$ Cedars-Sinai Medical Center, Los Angeles, CA, USA.

Received: 21 September 2016 Accepted: 14 January 2017

Published online: 04 March 2017

\section{References}

1. McAlindon T, Kissin E, Nasarian L, Ranganath V, Prakash S, Taylor M, et al. American College of Rheumatology report on reasonable use of musculoskeletal ultrasonography in rheumatology clinical practice. Arthritis Care Res. 2012;64:1625-40.

2. Colebatch AN, Edwards CJ, Ostergaard M, van der Heijde D, Balint PV, D-Agostino MA, et al. EULAR recommendations for the use of imaging of the joints in the clinical management of rheumatoid arthritis. Ann Rheum Dis. 2013;72:804-14.

3. Rowbotham E, Grainger A. Rheumatoid arthritis: Ultrasound vs. MRI. AJR. 2001;197:541-6.

4. Wakefield RJ, Balint PV, Szkudlarek M, Filippucci E, Backhaus M, D'Agostino $M A$, et al. Musculoskeletal ultrasound including definitions for ultrasonographic pathology. J Rheumatol. 2005;32:2485-7.

5. Backhaus M, Ohrndorf S, Kellner H, Strunk J, Backhaus TM, Hartung W, et al. Evaluation of a novel 7-joint ultrasound score in daily rheumatologic practice: a pilot project. Arthritis Rheum. 2009;61:1194-201.

6. Hama M, Uehara T, Takase K, Ihata A, Ueda A, Takeno M, et al. Power Doppler ultrasonography is useful for assessing disease activity and predicting joint destruction in rheumatoid arthritis patients receiving tocilizumab_-preliminary data. Rheumatol Int. 2012:32:1327-33.

7. Taylor PC, Steuer A, Gruber J, McClinton C, Cosgrove DO, Blomley MJ, et al. Ultrasonographic and radiographic results from a two-year controlled trial of immediate or one year delayed addition of infliximab to ongoing methotrexate therapy in patients with erosive early rheumatoid arthritis. Arthritis Rheum. 2006;54:47-53.

8. Naredo E, Bonilla G, Gamero F, Uson J, Carmona L, Laffon A. Assessment of inflammatory activity in rheumatoid arthritis: a comparative study of clinical evaluation with grey scale and power Doppler ultrasonography. Ann Rheum Dis. 2005;64:375-81.

9. Foltz V, Gandjbakhch F, Etchepare F, Rosenberg C, Tanguy ML, Rozenberg S, et al. Power Doppler ultrasound, but not low-field magnetic resonance imaging, predicts relapse and radiographic disease progression in rheumatoid arthritis patients with low levels of disease activity. Arthritis Rheum. 2012;64:67-76.

10. Giles JT, Ling SM, Ferrucci L, Bartlett SJ, Andersen RE, Towns M, et al. Abnormal body composition phenotypes in older rheumatoid arthritis patients: association with disease characteristics and pharmacotherapies. Arthritis Rheum. 2008:59:807-15.

11. Labitigan M, Bahce-Altuntas A, Kremer JM, Reed G, Greenberg JD, Jordan N, et al. Higher rates and clustering of abnormal lipids, obesity, and diabetes in psoriatic arthritis compared with rheumatoid arthritis. Arthritis Care Res. 2014;66:600-7.

12. Garcia-Poma A, Segami MI, Mora CS, Ugarte MF, Terrazas HN, Rhor EA, et al. Obesity is independently associated with impaired quality of life in patients with rheumatoid arthritis. Clin Rheumatol. 2007;26:1831-5.

13. Rodrigues AM, Reis JE, Santos C, Pereira MP, Loureiro C, Martins F, et al. A1.1 Obesity is a risk factor for worse treatment response in rheumatoid arthritis patients- results from reuma.pt. Ann Rheum Dis. 2014;73 Suppl 1:A1.

14. Klaasen R, Wijbrandts CA, Gerlag DM, Tak PP. Body mass index and clinical response to infliximab in rheumatoid arthritis. Arthritis Rheum. 2011;63:359-64.

15. Arnett FC, Edworthy SM, Bloch DA, McShane DJ, Fries JF, Cooper NS, et al. The American Rheumatism Association 1987 Revised criteria for the classification of rheumatoid arthritis. Arthritis Rheum. 1988;31:315-24.

16. Aletaha D, Neogi T, Silman A, Funovits J, Felson D, Binghman C, et al. 2010 Rheumatoid arthritis classification criteria: An Aerican College of Rheumatology/European League Against Rheumatism collaborative initative. Arthritis Rheum. 2010;62:2569-81.

17. Hammer HB, Bolton-King P, Bakkeheim V, Berg TH, Sundt E, Kongtorp AK, et al. Examination of intra and interrater reliability with a new ultrasonographic reference atlas for scoring of synovitis in patients with rheumatoid arthritis. Ann Rheum Dis. 2011;70:1995-8.

18. Sandberg MEC, Bengtsson C, Kallberg H, Wesley A, Klareskog L, Alfredsson L, et al. Overweight decreases the chance of achieving good response and low disease activity in early rheumatoid arthritis. Ann Rheum Dis. 2014;73:2029-33.

19. Smolen J, Aletaha A, Bijlsma J, Breedveld FC, Boumpas D, Burmester G, et al. Treating rheumatoid arthritis to target: recommendations of an international task force. Ann Rheum Dis. 2010;69:631-37.

20. Saunders SA, Capell HA, Stirling A, Vallance R, Kincaid W, McMahon AD, et al. Triple Therapy in early active rheumatoid arthritis: a randomized, singleblind, controlled trial comparing step-up and parallel treatment strategies. Arthritis Rheum. 2008;58:1310-7.

21. Grigor C, Capell H, Stirling A, McMahon AD, Lock P, Vallance R, et al. Effect of a treatment strategy of tight control for rheumatoid arthritis (the TICORA study): a single-blind randomized controlled trial. Lancet. 2004;364:263-9.

22. Pollard LC, Kingsley GH, Choy EH, Scott DL. Fibromyalgic rheumatoid arthritis and disease assessment. Rheumatology. 2010;49:424-8.

23. Ranzolin A, Brenol JC, Bredemeier M, Guarienti J, Rizzatti M, Feldman D, et al. Association of concomitant fibromyalgia with worse Disease Activity Score $n 28$ joints, Health Assessment Questionnaire and Short Form 36 scores in patients with rheumatoid arthritis. Arthritis Rheum. 2009;61:794-800.

24. Heimans L, van den Broek M, le Cessie S, Siegerink B, Riyazi N, Han KH, et al. Association of high body mass index with decreased treatment response to combination therapy in recent-onset rheumatoid arthritis patients. Arthritis Care Res. 2013;65:1235-42.

25. Gremese E, Carletto A, Padovan M, Atzeni F, Raffeiner B, Giardina AR, et al. Obesity and reduction of the response rate to anti-tumor necrosis factor alpha in rheumatoid arthritis: an approach to a personalized medicine. Arthritis Care Res. 2013;65:94-100.

26. Ajeganova S, Andersson ML, Hafstrom I. Association of obesity with worse disease severity in rheumatoid arthritis as well as with comorbidities: a longterm followup from disease onset. Arthritis Care Res. 2013;65:78-87.

27. Stavropoulos-Kalinoglou A, Metsios GS, Panoulas VF, Nevill AM, Jamurtas AZ, Koutedakis $Y$, et al. Underweight and obese states both associate with worse disease activity and physical function in patients with established rheumatoid arthritis. Clinical Rheumatol. 2009;28:439-44.

28. Uutela T, Kautiainen H, Jarvenpaa S, Salomaa S, Hakala M, Hakkinen A. Waist circumference based abdominal obesity may be helpful as a marker for unmet needs in patients with RA. Scand Journal Rheumatol. 2014;43:279-85.

29. Baker J, George M, Baker D, Toedter G, Von Felt JM, Leonard MB. Associations between body mass, radiographic joint damage, adipokines and risk factors for bone loss in rheumatoid arthritis. Rheumatology. 2011;50:2100-7.

30. van der Helm-van Mil AHM, van der Kooij SM, Allaart CF, Toes REM, Huizinga TWJ. A high body mass index has a protective effect on the 
amount of joint destruction in small joints in early rheumatoid arthritis. Ann Rheum Dis. 2008;67:769-74.

31. Kaufmann J, Kielstein V, Killian S, Stein G, Hein G. Relation Between Body Mass Index and Radiological Progression in Patients with Rheumatoid Arthritis. J Rheumatol. 2003;30:2350-5.

32. Giles JT, Allisone M, Bingham III CO, Scott Jr WM, Bathon JM. Adiponectin is a mediator of the inverse association of adiposity with radiographic damage in rheumatoid arthritis. Arthritis Rheum. 2009;61:1248-56.

33. Baker J, Ostergaard M, Geroge M, Justine S, Emery P, Baker DG, et al. Greater Body mass independently predicts less radiographic progression on X-ray and MRI over 1-2 years. Ann Rheum Dis. 2014;73:1923-8.

34. Naredo E, Moller I, Cruz A, Carmona L, Garrido J. Power Doppler ultrasonographic monitoring of response to anti-tumor necrosis factor therapy in patients with rheumatoid arthritis. Arthritis Rheum. 2008;58:2248-56.

35. Haavardsholm EA, Aga A, Olsen IC, Lillegraven S, Hammer HB, Uhlig T, et al. Ultrasound in management of rheumatoid arthritis: ARCTIC randomized controlled strategy trial. BMJ. 2016;354:14205.

36. Dale J, Stirling A, Zhang R, Purves D, Foley J, Sambrook M, Conaghan PG, et al. Targeting ultrasound remission in early rheumatoid arthritis: the results of the TaSER study, a randomized clinical trial. Ann Rheum Dis. 2016;75:1043-50.

37. Taouli B, Guermazi A, Sack KE, Genant HK. Imaging of the hand and wrist in RA. Ann Rheum Dis. 2002;61:867-9.

38. Wittoek R, Jens L, Lambrecht V, Carron P, Verstraete K, Verbruggen G. Reliability and construct validity of ultrasound of soft tissues and destructive changes in erosive osteoarthritis of the interphalangeal finger joints: a comparison with MRI. Ann Rheum Dis. 2011;20:278-83.

39. Backhaus M, Kamradt T, Sandrock D, Loreck D, Fritz J, Wolf KJ, et al. Arthritis of the finger joints: a comprehensive approach comparing conventional radiography, scintigraphy, ultrasound, and contrast-enhanced magnetic resonance imaging. Arthritis Rheum. 1999;42:1232-45.

\section{Submit your next manuscript to BioMed Central and we will help you at every step:}

- We accept pre-submission inquiries

- Our selector tool helps you to find the most relevant journal

- We provide round the clock customer support

- Convenient online submission

- Thorough peer review

- Inclusion in PubMed and all major indexing services

- Maximum visibility for your research

Submit your manuscript at www.biomedcentral.com/submit 